\title{
Qualidade de biscoitos diet adicionados de farinha da casca do maracujá avaliados sensorialmente por diabéticos
}

\section{Quality of diet cookies added of passion fruit peel flour (Passiflora edulis) sensorially evaluated by diabetic}

\author{
Ariany Dágma Batista Roque Santiago* \\ Janilson Avelino da Silva ** \\ Maria Lúcia da Conceição *** \\ Jailane de Souza Aquino ***
}

\begin{abstract}
Resumo
O objetivo deste trabalho foi elaborar biscoitos diet adicionados de farinha da casca do maracujá para avaliação físicoquímica, microbiológica e sensorial por portadores de diabetes. Foram formulados três tipos de biscoitos: um controle sem farinha da casca de maracujá (B1) e dois experimentais adicionados, respectivamente de 7 \% (B2) e 10 \% (B3) de farinha da casca de maracujá. Inicialmente foram realizadas análises físico-químicas e microbiológicas. O teste sensorial foi realizado por 87 portadores de diabetes tipo II recrutados em Unidades de Saúde da Família em João Pessoa - PB. A farinha do maracujá apresentou 23,58 \% de fibras e 4,32 \% de minerais. Os biscoitos adicionados de farinha da casca de maracujá apresentaram os maiores teores de minerais (entre 2,90 e 2,95 \%) e fibras (3,20 e 3,65 \%), e o biscoito B3 apresentou maior teor proteico (15,5\%). Todos os biscoitos apresentaram qualidade microbiológica desejável. As notas sensoriais variaram entre "gostei ligeiramente" e "gostei moderadamente" para todos os atributos avaliados, não havendo diferença estatística entre a intenção de compra e a preferência entre os biscoitos. O aproveitamento da casca do maracujá adicionada em biscoitos pode ser uma alternativa viável para diversificar os alimentos existentes no mercado, voltados para os portadores de diabetes.
\end{abstract}

Palavras-chave: Aceitação sensorial. Alimentos Diet. Diabetes. Processamento.

\begin{abstract}
The aim of this study was to develop to develop diet cookies added of passion fruit peel flourfor physicochemical, microbiological and sensory evaluation by patients with diabetes. Three types of cookies were formulated: control without passion fruit peel flour (B1) and two experimental cookies respectively added of $7 \%$ (B2) and 10 \% (B3) passion fruit peel flour. Initially, physicochemical and microbiological analyses were performed. The sensory test was performed by 87 patients with type II diabetes recruited from the Family Health Units of João Pessoa - PB. The flour of passion fruit peel showed $23.58 \%$ fiber and $4.32 \%$ minerals. Cookies added of passion fruit peel flour showed higher mineral $(2.90$ and $2.95 \%$ ) and fiber contents (3.20 and $3.65 \%$ ), and biscuit B3 showed higher protein content (15.5\%). All cookies showed desirable microbiological quality. The sensory scores ranged from "liked slightly" and "liked moderately" for all attributes evaluated, with no statistical difference between purchase intention and preference between cookies. The addition of passion fruit peel to cookies can be a viable alternative to diversify existing foods on the market aimed at people with diabetes.
\end{abstract}

Keywords: Sensory acceptance. Diet foods. Diabetes. Processing.

DOI: $10.15343 / 0104-7809.20164003362371$

\footnotetext{
*Serviço Nacional de Aprendizagem Industrial - SENAI. Campina Grande - PB, Brasil.

**Faculdades Integradas de Patos. Patos - PB, Brasil

***Universidade Federal da Paraíba (UFPB). João Pessoa - PB, Brasil. E-mail lalaaquino@hotmail.com

Os autores declaram não haver conflito de interesses.
} 


\section{INTRODUÇÃo}

O Brasil e outros países têm passado por processos de transição demográfica, epidemiológica e principalmente nutricional, durante as últimas décadas. Essas mudanças produzem importantes alterações no perfil de doenças da população causando o aumento significativo de doenças crônicas não transmissíveis, como o diabetes mellitus que é uma doença crônica que está afetando a população de forma crescente, tornando-se um sério problema de Saúde Pública ${ }^{1,2}$.

As doenças crônicas não transmissíveis são influenciadas, principalmente, por hábitos alimentares inadequados e um estilo de vida sedentário ${ }^{1}$. A maior incidência destas doenças deve-se, em grande parte, à redução do consumo de fibras na dieta ${ }^{1,3}$.

As fibras alimentares devem estar presentes, especialmente, na dieta de portadores de diabetes, tendo em vista que reduzem os níveis de hemoglobina glicada, melhoram a resposta glicêmica e as concentrações de insulina prandial ${ }^{4,5}$. Ademais, a fibra alimentar pode ser utilizada no enriquecimento de produtos ou como ingredientes, pois sua composição de polissacarídeos, lignina, oligossacarídeo e amido resistente, dentre outras substâncias, conferem diferentes propriedades funcionais aplicáveis à indústria de alimentos, podendo ser aproveitada na produção de diferentes

\section{MÉTODOS}

Os biscoitos diet foram elaborados no laboratório de Técnica e Dietética da Universidade Federal da Paraíba - UFPB. Foram elaboradas três formulações, uma formulação controle de biscoito diet não adicionada de farinha da casca do maracujá (B1) e duas formulações experimentais adicionadas de farinha da casca de maracujá, nas concentrações de $7 \%$ (B2) e $10 \%$ (B3) em substituição parcial da farinha de trigo devido ao alto teor de fibras presentes nesta farinha, com finalidade de reduzir o índice glicêmico nestes produtos. produtos como bebidas, sobremesas, derivados do leite, biscoito, massas e pães ${ }^{3}$.

Uma alternativa que vem crescendo desde o início da década de 1970 consiste no aproveitamento de resíduos, principalmente das cascas de frutas, como fonte de fibras a serem adicionadas aos alimentos ${ }^{6}$. Dentre estas, tem-se a farinha da casca do maracujá que, nos últimos anos, tem sido estudada devido às diversas propriedades hipoglicemiantes, anti-hipertensivas, antioxidante, entre outras propriedades $^{7-9}$.

A importância de tal temática é demonstrada pelo fato de promover a utilização de partes dos alimentos com potencial valor nutricional que, geralmente, não são aproveitados e podem agregar valor aos resíduos, como da casca do maracujá. Isto, além da necessidade de suprir a demanda dos consumidores, principalmente dos portadores de diabetes por alimentos diet que, muitas vezes, é limitada em função do custo e das características sensoriais destes alimentos.

Neste sentido, este estudo objetivou desenvolver biscoitos diet adicionados de diferentes concentrações de farinha da casca do maracujá para avaliação físico-química, microbiológica e sensorial, sendo esta última realizada por grupos de indivíduos portadores de diabetes.
Os biscoitos foram formulados de acordo com a metodologia adaptada de Alfenas et al. ${ }^{10}$, utilizando os seguintes ingredientes: farinha de trigo integral, farinha de trigo comum, farinha da casca de maracujá comercial, amido de milho, adoçante dietético para uso culinário (ciclamato de sódio e sacarina sódica), óleo de soja, leite em pó desnatado, leite pasteurizado, ovo, fermento em pó, sal e lecitina de soja (Tabela 1), tais ingredientes foram adquiridos em supermercados da cidade de João PessoaPB. 
Tabela 1 - Ingredientes de formulações de biscoitos diet adicionados de farinha de casca de maracujá em diferentes concentrações. João Pessoa-PB, 2014.

\begin{tabular}{lcccccc}
\hline \multicolumn{1}{c}{ Ingredientes } & \multicolumn{2}{c}{ B1 } & \multicolumn{2}{c}{ B2 } & \% & B3 \\
\hline Farinha de trigo integral & 27,17 & 219 & 27,17 & 219 & 27,17 & 219 \\
Farinha de trigo comum & 18,61 & 150 & 14,90 & 120 & 13,03 & 105 \\
Farinha da casca do maracujá & 0 & 0 & 3,72 & 30 & 5,60 & 45 \\
Amido de Milho & 8,31 & 67 & 8,31 & 67 & 8,31 & 67 \\
Edulcorantes ciclamato de sódio & 2,00 & 16 & 2,00 & 16 & 2,00 & 16 \\
e sacarina sódica & & & & & & \\
Óleo de soja & 7,00 & 56 & 7,00 & 56 & 7,00 & 56 \\
Leite em pó desnatado & 4,47 & 36 & 4,47 & 36 & 4,47 & 36 \\
Leite pasteurizado & 15,00 & 120 & 15,00 & 120 & 15,00 & 120 \\
Ovo & 15,00 & 120 & 15,00 & 120 & 15,00 & 120 \\
Fermento em pó & 1,24 & 10 & 1,24 & 10 & 1,24 & 10 \\
Sal & 0,12 & 1 & 0,12 & 1 & 0,12 & 1 \\
Lecitina de soja & 1,36 & 11 & 1,36 & 11 & 1,36 & 11 \\
\hline
\end{tabular}

Fonte: Adaptado de Alfenas et al. ${ }^{10}$

B1 - biscoito diet controle (0 \% de farinha de casca de maracujá);

B2 - biscoito diet adicionado de $7 \%$ de farinha de casca de maracujá;

B3 - biscoito diet adicionado de $10 \%$ de farinha de casca de maracujá.

Os biscoitos foram processados de acordo com as seguintes etapas: pesagem dos ingredientes, mistura e descanso da massa, formação do biscoito, cozimento, resfriamento, empacotamento e armazenamento. Inicialmente, todos os ingredientes foram pesados em balança digital (Filizola ${ }^{\circledR}$, São Paulo, Brasil). Em seguida, foram misturados em um recipiente, os ovos, o sal, o óleo, o adoçante, o fermento, o leite e a lecitina, até a formação de um creme. Depois, foram adicionadas à mistura, as farinhas e o amido de milho, amassando bem até formar uma massa homogênea. Após esta mistura, a massa foi coberta por filme plástico, permanecendo em descanso por 5 minutos. Posteriormente, as massas foram modeladas com auxílio de um rolo de massas e cortadores de biscoito e colocadas em formas, sendo cobertas por papel vegetal.

Após a modelagem, os biscoitos foram assados em forno convencional pré-aquecido a $180^{\circ} \mathrm{C}$ por 16 minutos. Depois de assados, os biscoitos foram retirados do forno e deixados em temperatura ambiente por 30 minutos para, em seguida, serem acondicionados em embalagem de polietileno lisa e transparente. Os produtos foram mantidos em condições normais de armazenamento, à temperatura ambiente, por no máximo dois dias até utilização.

A composição centesimal da farinha da casca do maracujá e dos biscoitos foi realizada em triplicata a partir das análises de umidade, cinzas, proteínas, lipídios ${ }^{11}$ e fibra alimentar pelo método gravimétrico não enzimático ${ }^{12}$ além dos carboidratos que foram calculados por diferença ${ }^{13}$. O valor energético das formulações foi calculado, utilizando os coeficientes de Atwater ${ }^{13}$.

Os biscoitos foram analisados microbiologicamente, em triplicata, e de acordo com as análises recomendadas pela RDC n.12 de $2001^{14}$. Foi realizada pesquisa de Salmonella sp, a contagem de Estafilococos coagulase positiva pela técnica spread plate, bem como a contagem padrão de bactérias aeróbias mesófilas, realizada pela técnica pour plate. Os bolores e as leveduras foram quantificados pela técnica de espalhamento em superfície 
por semeadura. A enumeração de coliformes a $35{ }^{\circ} \mathrm{C}$ e coliformes termotolerantes a $45{ }^{\circ} \mathrm{C}$ foram estimadas pela técnica do Número Mais Provável (NMP).

As análises sensoriais foram realizadas durante o primeiro semestre de 2014, em salas com adequada luminosidade cedidas pelas Unidades de Saúde. Participaram da análise sensorial, indivíduos diabéticos que eram atendidos em 17 Unidades de Saúde pela Estratégia Saúde da Família (ESF), estando localizadas no Distrito Sanitário III, do município de João Pessoa-PB, o qual é considerado um dos distritos de maior abrangência em termos de atendimento populacional, território e de Unidades de Saúde da Família - USF. Foram selecionados indivíduos diabéticos, de ambos os sexos, com idades variando entre 35 e 70 anos, em que a quantidade de voluntários participantes foi de acordo com a demanda de cada USF, totalizando 87 participantes.

O presente estudo foi realizado mediante autorização institucional da Secretaria de Saúde do município de João Pessoa-PB e aprovação do Comitê de Ética em Pesquisa do Centro de Ciências da Saúde da Universidade Federal da Paraíba- UFPB sob o no 341/2011. Todos os participantes que aceitaram participar da pesquisa assinaram o Termo de Consentimento Livre e Esclarecido (TCLE) conforme preconizado na Resolução n.466/2012 do Conselho Nacional de Saúde ${ }^{15}$.

As variáveis estudadas em relação ao perfil dos indivíduos foram: idade, sexo, grau de escolaridade e frequência de consumo de biscoitos. Os instrumentos utilizados na coleta de dados foram um formulário para análise sensorial do alimento em questão e um questionário com os dados gerais dos usuários.

Os 87 participantes portadores de diabetes que se disponibilizaram a participar do estudo realizaram a análise sensorial das três formulações de biscoitos diet em única sessão, nas respectivas USF e foram recrutados com base na frequência de consumo de biscoitos, segundo os níveis de ingestão: alto (consumo diário); moderado (1 a 2 vezes por semana); baixo (quinzenalmente) e muito baixo (ocasionalmente).

As amostras de biscoitos, contendo 4 gramas cada, foram servidas simultaneamente em temperatura usual de consumo (ambiente), em pratos plásticos de cor branca, previamente codificadas com três dígitos numéricos aleatórios, acompanhadas do formulário de avaliação sensorial. Juntamente com as amostras foi oferecida, aos provadores, água à temperatura ambiente para ser consumida entre as avaliações.

No teste de aceitação foram avaliados os atributos sensoriais: aparência, aroma, textura, sabor e aceitação global, através da escala hedônica estruturada de nove pontos (1=desgostei extremamente; $5=$ =indiferente; $9=$ gostei extremamente) de acordo com Meilgaard et $\mathrm{al}^{16}$. O teste de preferência por ordenação foi realizado, bem como a avaliação da intenção de compra, categorizada em escala variando de: $1=$ Certamente não compraria; 2=Provavelmente não compraria; $3=$ Talvez comprasse, talvez não comprasse; $4=$ Provavelmente compraria e $5=$ Certamente compraria $^{16}$.

Os dados obtidos foram submetidos à análise de variância (ANOVA) e as médias comparadas pelo teste de Tukey a $5 \%$ de significância, utilizando o programa estatístico Sigma Stat, versão 3.5.

\section{RESULTADOS}

A farinha da casca de maracujá apresentou $7,21 \pm 0,10 \%$ de umidade, $4,32 \pm 0,15 \%$ de cinzas, $0,98 \pm 0,08 \%$ de proteínas, 0,93 $\pm 0,10$ $\%$ de lipídios, 23,58 0,16 \% de fibra alimentar.

$\mathrm{Na}$ Tabela 2 se encontram os dados da composição centesimal e do valor energético dos biscoitos diet controle (B1) e experimentais contendo, respectivamente, $7 \%$ (B2) e $10 \%$ (B3) da farinha de casca de maracujá.

Os biscoitos diet adicionados de farinha de casca de maracujá apresentaram menores teores de umidade e maiores teores de cinzas. A formulação de maior concentração de farinha de maracujá (B3) apresentou um maior teor de proteínas e fibra alimentar e menor valor energético $(p<0,05)$.

Detectaram-se valores $<3 \mathrm{NMP} / \mathrm{mL}$ (ausência) de coliformes totais e fecais, ausência 
na contagem de bolores e leveduras e bactérias aeróbias e mesófilas, bem como ausência em 25 g de Staphylococcus coagulase positiva e de Salmonella spp em todas as amostras analisadas (Tabela 3).

No recrutamento dos provadores foi verificada uma predominância do sexo feminino (71\%) e da faixa etária acima de 55 anos de idade $(72 \%)$, entre os portadores de diabetes participantes da pesquisa. Em relação ao nível de escolaridade, a maior parte dos voluntários cursou até o ensino fundamental incompleto (36\%) e ensino médio completo (18\%). Um total de $55 \%$ dos portadores de diabetes que realizaram os testes sensoriais relatou ter hábito de consumir biscoitos diariamente, caracterizando um alto consumo deste produto. $(p<0,05)$ farinha de casca de maracujá. João Pessoa-PB, 2014.
Não houve diferença estatística significativa $(p>0,05)$ entre as amostras, quanto aos atributos sensoriais avaliados de aparência, aroma, textura, sabor e aceitação global. No entanto, os biscoitos B3, de maior concentração de farinha da casca de maracujá (10\%), apresentaram a menor intenção de compra pelos portadores de diabetes (Tabela 4).

Na Tabela 5 observa-se a distribuição das notas sensoriais de acordo com a ordenação de preferência geral dos provadores. Verificou-se uma preferência sensorial semelhante entre as amostras de biscoito diet, ou seja, na soma das ordens não houve diferença significativa entre os biscoitos $(p>0,05)$ pelo teste de Friedman o que reforça os resultados dos testes de aceitação sensorial.

Tabela 2 - Composição centesimal e valor energético de biscoitos diet adicionados de diferentes concentrações de farinha de casca de maracujá. João Pessoa-PB, 2014.

\begin{tabular}{lccc}
\hline \multirow{2}{*}{\begin{tabular}{c} 
Parâmetros físico-químicos \\
\cline { 2 - 4 }
\end{tabular}} & \multicolumn{3}{c}{ Biscoitos } \\
\cline { 2 - 4 } & & B1 & B2 \\
\hline Umidade (\%) & $11,54 \mathrm{a} \pm 0,04$ & $9,82 \mathrm{~b} \pm 0,09$ & B3 \\
Cinzas (\%) & $2,68 \mathrm{~b} \pm 0,03$ & $2,90 \mathrm{a} \pm 0,03$ & $2,95 \mathrm{a} \pm 0,01$ \\
Proteínas (\%) & $13,85 \mathrm{~b} \pm 0,28$ & $14,01 \mathrm{~b} \pm 0,35$ & $15,50 \mathrm{a} \pm 0,08$ \\
Lipídios (\%) & $22,80 \mathrm{a} \pm 0,28$ & $19,65 \mathrm{~b} \pm 043$ & $16,00 \mathrm{c} \pm 0,28$ \\
Carboidratos (\%) & $46,25 \mathrm{c} \pm 0,64$ & $47,57 \mathrm{~b} \pm 0,90$ & $49,15 \mathrm{a} \pm 0,83$ \\
Fibra alimentar (\%) & $2,88 \mathrm{c} \pm 0,18$ & $6,05 \mathrm{~b} \pm 0,23$ & $7,05 \mathrm{a} \pm 0,19$ \\
Valor energético (kcal) & $457,12 \mathrm{a} \pm 4,60$ & $447,38 \mathrm{a} \pm 8,87$ & $354,40 \mathrm{~b} \pm 5,33$ \\
\hline
\end{tabular}

Médias \pm desvio-padrão com letras diferentes na mesma linha diferiram entre si pelo teste de Tukey

B1 - biscoito diet controle (0 \% de farinha de casca de maracujá);

B2 - biscoito diet adicionado de $7 \%$ de farinha de casca de maracujá;

B3 - biscoito diet adicionado de $10 \%$ de farinha de casca de maracujá.

Tabela 3 - Análises microbiológicas de biscoitos diet adicionados de diferentes concentrações de

\begin{tabular}{lccc}
\hline & \multicolumn{3}{c}{ Biscoitos } \\
\cline { 2 - 4 } Análises microbiológicas & B1 & B2 & B3 \\
\hline Bactérias aeróbias mesófilas & Ausência & Ausência & Ausência \\
Coliformes totais & Ausência $<3,0$ & Ausência $<3,0$ & Ausência $<3,0$ \\
\hline
\end{tabular}




\begin{tabular}{|c|c|c|c|}
\hline Coliformes fecais & $<3,0 \mathrm{NMP} / \mathrm{mL}$ & $<3,0 \mathrm{NMP} / \mathrm{mL}$ & $<3,0 \mathrm{NMP} / \mathrm{mL}$ \\
\hline Staphylococcus & Ausência & Ausência & Ausência \\
\hline Salmonella & Ausência & Ausência & Ausência \\
\hline Bolores e Leveduras & Ausência & Ausência & Ausência \\
\hline
\end{tabular}

B1 - biscoito diet controle (0 \% de farinha de casca de maracujá);

B2 - biscoito diet adicionado de $7 \%$ de farinha de casca de maracujá;

B3 - biscoito diet adicionado de $10 \%$ de farinha de casca de maracujá

Tabela 4 - . Valores médios de aceitação sensorial e intenção de compra de biscoitos diet adicionados de diferentes concentrações de farinha de casca de maracujá analisados. João Pessoa-PB, 2014.

\begin{tabular}{lccc}
\hline \multicolumn{1}{c}{ Atributos sensoriais* } & \multicolumn{3}{c}{ Biscoitos } \\
\cline { 2 - 4 } & B1 & B2 & B3 \\
\hline Aparência & $7,03 \mathrm{a} \pm 1,51$ & $7,38 \mathrm{a} \pm 1,35$ & $7,00 \mathrm{a} \pm 1,50$ \\
Aroma & $6,41 \mathrm{a} \pm 1,87$ & $6,79 \mathrm{a} \pm 1,73$ & $6,39 \mathrm{a} \pm 1,74$ \\
Sabor & $7,28 \mathrm{a} \pm 1,67$ & $7,14 \mathrm{a} \pm 1,86$ & $6,71 \mathrm{a} \pm 1,95$ \\
Textura & $6,72 \mathrm{a} \pm 1,82$ & $6,70 \mathrm{a} \pm 1,88$ & $6,48 \mathrm{a} \pm 2,00$ \\
Avaliação Global & $7,69 \mathrm{a} \pm 1,76$ & $7,81 \mathrm{a} \pm 1,53$ & $7,49 \mathrm{a} \pm 1,78$ \\
Intenção de Compra** & $4,16 \mathrm{a} \pm 1,19$ & $3,89 \mathrm{a} \pm 1,35$ & $3,37 \mathrm{~b} \pm 1,41$ \\
\hline
\end{tabular}

$\mathrm{n}=87$

*Escala hedônica: ( $1=$ desgostei extremamente; $9=$ gostei extremamente)

** Escala de intenção de compra ( $1=$ certamente não compraria; $5=$ certamente compraria)

Médias com letras diferentes na mesma linha indicam diferença entre si pelo teste de Tukey $(p<0,05)$ B1 - biscoito diet controle (0 \% de farinha de casca de maracujá);

B2 - biscoito diet adicionado de $7 \%$ de farinha de casca de maracujá;

B3 - biscoito diet adicionado de $10 \%$ de farinha de casca de maracujá.

Tabela 5 - Distribuição das notas da ordenação de preferência geral dos julgadores (n=87) para biscoitos diet adicionados de diferentes concentrações de farinha de casca de maracujá. João PessoaPB, 2015.

\begin{tabular}{|c|c|c|c|c|}
\hline \multirow[t]{2}{*}{ Biscoitos } & \multicolumn{3}{|c|}{ Número de Provadores por Ordem* } & \multirow[t]{2}{*}{ Somas das ordens ${ }^{* *}$} \\
\hline & 1 & 2 & 3 & \\
\hline B1 & 31 & 23 & 32 & $173^{\mathrm{a}}$ \\
\hline B2 & 21 & 32 & 33 & $184^{\mathrm{a}}$ \\
\hline B3 & 35 & 30 & 21 & $158^{\mathrm{a}}$ \\
\hline
\end{tabular}

* Notas de ordenação preferência: 1 = menos preferido, 3 = mais preferido

** Soma das ordens de cada amostra $=\left(1 \times \mathrm{n}^{\circ}\right.$ de provadores $)+\left(2 \times \mathrm{n}^{\circ}\right.$ de provadores $)+\left(3 \times \mathrm{n}^{\circ}\right.$ de provadores). A: indica que não houve diferença significativa entre os biscoitos $(p<0,05)$ pelo teste de Friedman

B1 - biscoito diet controle (0 \% de farinha de casca de maracujá);

B2 - biscoito diet adicionado de $7 \%$ de farinha de casca de maracujá;

B3 - biscoito diet adicionado de $10 \%$ de farinha de casca de maracujá. 


\section{DISCUSSÃo}

Os resultados da composição centesimal da farinha da casca de maracujá comercial estão próximos aos determinados por Uchoa et al. ${ }^{17}$ para a farinha do resíduo do maracujá, exceto a quantidade de fibra alimentar que foi superior, de fibras ${ }^{18}$.

A umidade dos biscoitos diet variou de 9,35 a 11,54\%, atendendo ao Regulamento Técnico para Produtos de Cereais, Amidos, Farinhas e Farelos ${ }^{19}$, que estipula valor máximo de $15 \%$ de umidade para este produto. O percentual de umidade foi menor no biscoito B3, possivelmente porque a farinha da casca de maracujá apresenta maior teor de fibras do que a farinha de trigo branca e a integral e não contém glúten, sendo este componente o responsável pela maior retenção de umidade nas farinhas de trigo ${ }^{20}$. No entanto, Santana et al. ${ }^{21}$ observaram que a incorporação de fécula de mandioca e farinha da casca de maracujá a biscoitos resultou no aumento do percentual de umidade, quando comparado ao biscoito padrão. Os biscoitos analisados no presente estudo apresentaram percentual de umidade acima dos relatados por Uchoa et al. ${ }^{17}$ que foi de 4,9\% para cookies adicionados de pó de caju e 4,1\% para os contendo pó de goiaba.

Os percentuais de cinzas aumentaram conforme a adição da farinha da casca de maracujá, resultado semelhante foi encontrado por Maciel $^{22}$ adicionando farinha de linhaça em biscoitos cream cracker. Finco et al..23 elaborando biscoitos contendo $10 \%$ de farinha de berinjela obtiveram 1,01\% de cinzas, valor inferior ao obtido no presente trabalho.

O aumento da concentração de farinha da casca de maracujá aumentou o percentual de proteínas dos biscoitos diet, entretanto, em estudo realizado por Uchoa et al. ${ }^{17}$, o teor de proteínas não resultou em diferença significativa após a adição de pó de caju à formulação de biscoitos tipo cookies. A Ingestão Diária Recomendada (IDR) de proteínas para adultos diabéticos deve estar entre 15 e $20 \%$ das calorias totais da dieta, caso a função renal esteja normal ${ }^{24}$, levando-se em consideração uma porção de 32 g que equivale a 4 unidades

de cookies, os biscoitos diet B2 e B3 contribuem respectivamente com 0,90 e 0,99\% das calorias provenientes de proteínas, considerando uma dieta de 2000 Kcal.

O teor de lipídios e o valor calórico foram semelhantes entre os diferentes biscoitos analisados, sendo este um dado importante, pois sugere que a farinha de casca de maracujá não incorporou um adicional de gordura aos biscoitos como ocorre com outras farinhas, a exemplo da farinha de linhaça (Linum ussitatissimo L.) adicionada a biscoitos tipo Cracker ${ }^{22}$.

Os biscoitos diet apresentaram teor de carboidratos entre 46,25 g/100g e 49,15 $\mathrm{g} / 100 \mathrm{~g}$, sendo estes valores inferiores aos obtidos por Santana et al. ${ }^{21}$ para biscoitos adicionados de farinha de maracujá e fécula de mandioca e por Mauro et al. ${ }^{25}$ para cookies enriquecidos com talo de couve e de espinafre. Os carboidratos devem perfazer da ingestão energética total, 45 a $60 \%$ das calorias, para um adulto que receberia $2000 \mathrm{Kcal} / \mathrm{dia}^{24}$. Na prática, o consumo de quatro biscoitos diet (32 g) produzidos com farinha da casca do maracujá perfazem em média 5,63\% das IDR de carboidratos para diabéticos, calculado com base em $55 \%$ de carboidratos em uma dieta de 2000 Kcal. Importante salientar que o consumo de carboidratos complexos deve ser priorizado pela população em geral e principalmente pelos portadores de diabetes, assim como a redução do consumo de carboidratos refinados ${ }^{26}$, e neste sentido, os biscoitos diet adicionados de farinha da casca de maracujá aqui elaborados podem contribuir para esta recomendação.

O teor de fibras alimentares aumentou nos biscoitos diet à medida que foi incorporada a farinha da casca do maracujá, sendo as formulações B2 e B3 consideradas fontes de fibras, por terem atingido o mínimo $3 \mathrm{~g}$ fibras/100 g deste produto, preconizado pela Agência Nacional de Vigilância Sanitária - ANVISA $^{18}$, além de terem apresentado mais do que o dobro de fibra alimentar do biscoito controle (B1). Sendo o teor de fibras dos biscoitos adicionados de farinha da casca do maracujá superiores aos de biscoitos adicionados de albedo de laranja avaliados por Santos et al. ${ }^{27}$, que apresentou uma porcentagem de $2,11 \%$ de fibras em biscoitos com adição de 
7,5\% de albedo de laranja, não podendo ser considerado fonte de fibras. O percentual de fibras dos biscoitos B2 e B3 foram superiores ao obtido por Ishimoto et al. ${ }^{7}$ que obtiverem 3,24 $\%$ nos biscoitos adicionados de $30 \%$ da farinha da casca de maracujá.

Independente da quantidade sabe-se que as fibras auxiliam na diminuição do Índice Glicêmico (IG) dos alimentos devido à gelatinização incompleta e retrogradação do amido contra a ação das enzimas amilases no amido $^{28}$. O IG representa o aumento da área sob a curva da resposta glicêmica produzida por um determinado alimento, em relação à mesma quantidade (50 g) de carboidrato disponível de um produto padrão ${ }^{29}$, indicando que a presença de fibras em biscoitos diet torna-se uma ferramenta importante na terapia nutricional de diabéticos.

As amostras se encontram dentro dos padrões microbiológicos estabelecidos pela RDC $n^{-}$12, de 02 de janeiro de 200114, indicando que as preparações estavam próprias para consumo humano e que o processo de elaboração seguiu as normas de Boas Práticas de Fabricação (BPF) recomendadas pela Resolução RDC n 216, de 15 de setembro de $2004{ }^{30}$. Assegurar a qualidade microbiológica dos biscoitos diet, propicia maior segurança no consumo destes alimentos principalmente para os indivíduos que apresentam algum tipo de patologia, neste caso os diabéticos, tendo em vista que o consumo de alimentos contaminados por microrganismos pode agravar ainda mais o quadro clínico destes indivíduos.

A maior parte dos portadores de diabetes que participaram deste estudo era do sexo feminino com faixa etária acima de 55 anos de idade, apresentando perfil amostral semelhante ao do estudo de Fontbonne et al. ${ }^{31}$ e Carvalho et al. ${ }^{32}$.

Os biscoitos diet apresentaram boa aceitação sensorial, não havendo diferença estatística $(p>0,05)$ para nenhum dos atributos sensoriais avaliados, demonstrando que a adição de farinha da casca de maracujá em diferentes percentuais não influenciou a aceitação destes biscoitos e que a mesma pode ser utilizada como matéria-prima alternativa para a elaboração de biscoitos.

As notas para todos os atributos se encontraram entre "gostei ligeiramente" e "gostei moderadamente", refletindo uma qualidade global semelhante entre os biscoitos, com médias expressas como "gostei moderadamente". Para a avaliação global, apesar de considerar-se a homogeneidade de importância dos atributos agregados como, aparência, aroma, textura e sabor, pode haver uma hierarquia na importância relativa da contribuição de cada atributo.

A análise sensorial de biscoitos enriquecidos com aveia e farinha de bagaço de uva obteve médias entre 6,86 e 7,30 como avaliação global, valores estes próximos aos encontrados no presente estudo. A combinação de farinhas não convencionais com a farinha de trigo melhora a qualidade nutricional de biscoitos, podendo melhorar a palatabilidade, deixando-os mais aceitos pelos consumidores ${ }^{33}$.

Um estudo em que se avaliou a aceitação sensorial da adição de farinha de uva em biscoitos demonstrou que a adição de $10 \%$ desta farinha não gerou diferença estatística em relação ao biscoito controle quanto à maioria dos atributos sensoriais investigados ${ }^{34}$.

Em outro estudo com adição de farinha de baru, quando o atributo sabor foi avaliado observou-se que não houve diferença $(p>0,05)$ quanto à preferência entre a amostra controle e os demais tratamentos dos biscoitos, se assemelhando a esta investigação. A adição de $8 \%$ de farinha de amêndoa de baru, em substituição a quantidades iguais de farinha de trigo e fécula de mandioca, não afetou a aparência, o sabor e a textura de biscoitos cookie ${ }^{35}$.

Handa et al. ${ }^{4}$ avaliando a adição de fibras substituindo o açúcar em biscoitos não observaram diferença estatística $(p>0,05)$ quanto aos aspectos sensoriais de cor, sabor, textura, aparência e aceitação global em até 40 \% de substituição. No entanto, Ajila et al. ${ }^{36}$ observaram que a incorporação de $20 \%$ de farinha de casca de manga resultou num ligeiro sabor amargo nos biscoitos. Em outra investigação, a incorporação de farinha de resíduos de acerola em substituição da farinha de trigo promoveu uma menor aceitação dos biscoitos em comparação à formulação controle $^{37}$, indicando a importância da seleção da quantidade de farinha de resíduos a ser 
substituída em produtos alimentícios, sob pena de reduzir a aceitação destes.

A maior parte dos provadores julgou que "provavelmente comprariam" os biscoitos diet controle (B1) e com $7 \%$ de farinha de casca de maracujá (B2), expressando maior intenção de compra para estes produtos, enquanto que "talvez comprasse, talvez não comprasse" o biscoito diet B3 (10\% de farinha da casca do maracujá), avaliado em escala de 5 pontos. Logo, as médias de intenção de compra para os biscoitos diet, foram superiores as obtidas por Santana et al. ${ }^{18}$ que foram expressas por "talvez comprasse/talvez não comprasse".

\section{CONCLUSÃO}

Os biscoitos diet adicionados de farinha da casca de maracujá apresentaram valores expressivos de nutrientes, com destaque para o elevado teor de minerais, fibra alimentar e proteínas, além do baixo valor energético. Os biscoitos diet apresentaram qualidade microbiológica desejável bem como aceitação sensorial e preferências semelhantes, para todos os tratamentos realizados. Diante do exposto, o maracujá é uma fruta de fácil acesso e o aproveitamento de sua casca na produção de farinha pode ser uma alternativa viável para o enriquecimento de biscoitos e demais produtos de panificação, diversificando os produtos para fins especiais existentes no mercado e reduzindo o desperdício desse resíduo no agronegócio.

AGRADECIMENTOS: À Secretaria de Saúde do Município de João Pessoa-PB, às Unidades de Saúde pela Estratégia Saúde da Família (ESF) e aos voluntários participantes deste estudo.

\section{REFERÊNCIAS}

1. Queiroz MSR, Janebro DI, Cunha MAL, Medeiros JS, Sabaa-Srur AU, Diniz MFFM, et al. Effect of the yellow passion fruit peel flour (Passiflora edulis f. flavicarpa Deg.) in insulin sensitivity in type 2 diabetes mellitus patients. Nutrition J. 2012; 11(89): 2-7.

2. Guimarães FPM, Takayanagui AMM. Orientações recebidas do serviço de saúde por pacientes para o tratamento do portador de diabetes mellitus tipo 2. Rev Nutr. 2002; 15(1):37-44.

3. Souza MWS, Ferreira TBO, Vieira IFR. Composição Centesimal e Propriedades Funcionais tecnológicas da Farinha da Casca do Maracujá. Alim Nutr. 2008; 19(1):33-36.

4. Handa C, Goomer S, Siddhu A. Physicochemical properties and sensory evaluation of fructoligosaccharide enriched cookies. J Food Sci Technol. 2012; 49(2):192-199.

5. Silva FM, Kramer CK, de Almeida JC, Steemburgo T, Gross JL, Azevedo MJ. Fiber intake and glycemic control in patients with type 2 diabetes mellitus: a systematic review with meta-analysis of randomized controlled trials. Nutr Rev. 2013; 71(12): $790-801$.

6. Oliveira LF, Nascimento MRF, Borges SV, Ribeiro PCN, Ruback VR. Aproveitamento alternativo da casca do maracujá-amarelo (Passiflora edulis F. Flavicarpa) para produção de doce em calda. Cienc Tecnol Alim. 2002; 3(22): 259-262.

7. Ishimoto FY, Harada AI, Branco IG, Conceição WAS, Coutinho MR. Aproveitamento Alternativo da Casca do Maracujá-Amarelo (Passiflora edulis f. Var .flavicarpa Deg.) para Produção de Biscoitos. Rev Cienc Exat Nat. 2007; 9(2): 279-292.

8. Espirito-Santo AP, Lagazzo A, Sousa ALOP, Perego P, Converti A, Maricê NO. Rheology, spontaneous whey separation, microstructure and sensorial characteristics of probiotic yoghurts enriched with passion fruit fiber. Food Res Int. 2013; 50 (1): 224-231

9. López-Vargas JH, Fernández-López J, Pérez-Álvarez JA, Viuda-Martos M. Chemical, physico-chemical, technological, antibacterial and antioxidant properties of dietary fiber powder obtained from yellow passion fruit (Passiflora edulis var. flavicarpa) co-products. Food Res Int. 2013; 51 (2):756-763.

10. Alfenas RCG, Queiroz, VMV, Bittencourt MCB, Silva MMSS. Diabetes - dieta e receitas especiais. $3^{\underline{a}}$ ed. Viçosa: Editora UFV; 2008. 11. Association of Official Analytical Chemists-AOAC. Official methods of analysis of the Association of Official Analytical Chemists. 17th ed. Washington: AOAC; 2002.

12. Guerra NB, David PRBS, Melo DD, Vasconcelos ABB, Guerra MRM. Modificações do método gravimétrico não enzimático para determinar fibra alimentar solúvel e insolúvel em frutos. Rev. Nutr. 2004; 17(1): 45-52.

13. Brasil. Agência Nacional de Vigilância Sanitária. Resolução RDC n 360, de 23 de dezembro de 2003. Regulamento Técnico sobre Rotulagem Nutricional de Alimentos Embalados, tornando obrigatória a rotulagem nutricional. Diário Oficial da União. 2003 dez 26; Seção 1.

14. Brasil. Agência Nacional de Vigilância Sanitária. Resolução RDC no 12, de 2 de janeiro de 2001. Regulamento técnico sobre os padrões microbiológicos para alimentos. Diário Oficial da União. 2001 jan 2; Seção 1.

15. Conselho Nacional de Saúde (CNS). [acesso 2016 set 30]. Disponível em: < http://conselho.saude.gov.br/resolucoes/2012/ 
Reso466.pdf>

16. Meilgaard M, Civille GV, Carr BT. Consumer test and in-house panel acceptance tests. Sensory Evaluation Techniques. Florida: CRC Press Inc; 1988.

17. Uchoa AMA, Costa JMC, Maia GA, Meira TR, Sousa PHM, Brasil IM. Formulation and Physicochemical and Sensorial Evaluation of Biscuit-Type Cookies Supplemented with Fruit Powders. Plant Foods Hum Nutr. 2009; 64 (2): 153-159.

18. Brasil. Ministério da Saúde. Portaria no 31 de 13 de Janeiro de 1998 - Regulamento Técnico referente a Alimentos Adicionados de Nutrientes Essenciais. Diário Oficial da União. 1998 jan 16; Seção 1.

19. Brasil. Agência Nacional de Vigilância Sanitária. Resolução RDC n²63, de 22 de setembro de 2005. Regulamento Técnico para Produtos de Cereais, Amidos, Farinhas e Farelos. Diário Oficial da União. 2005 set 23; Seção 1.

20. Fernandes AF, Pereira J, Germani R, Oiano-Neto J. Efeito da substituição parcial da farinha de trigo por farinha de casca de batata (Solanum Tuberosum Lineu). Ciênc Tecnol Aliment. 2008: 28: 56-65.

21. Santana FC, Silva JV, Santos AJAO, Alves AR, Wartha ERSA, Marcellini OS, et al. Desenvolvimento de biscoito rico em fibras elaborado por substituição parcial da farinha de trigo, por farinha da casca do maracujá amarelo (Passiflora edulis flavicarpa) e fécula de mandioca (Manihot esculenta crantz). Alim Nutr. 2011; 22 (3): 391-399.

22. Maciel LMB, Pontes DF, Rodrigues MCP. Efeito da adição de farinha de linhaça no processamento de biscoito tipo Cracker. Alim Nutr. 2008; 19 (4):385-392.

23. Finco AMO, Bezerra JRMV, Rigo M, Córdova KRV. Elaboração de biscoitos com adição de farinha de berinjela. Rev Bras Tecnol Agro. 2009; 3(1); 49-59.

24. Sociedade Brasileira de Diabetes-SBD. Diretrizes da Sociedade Brasileira de Diabetes (2015-2016). Brasil: A.C. Farmacêutica, 2016. [acesso 2016 out 01]. Disponível em: < http://www.diabetes.org.br/sbdonline/images/docs/DIRETRIZES-SBD-2015-2016.pdf>

25. Mauro AK, Silva VLM, Freitas MCJ. Caracterização física, química e sensorial de cookies confeccionados com Farinha de Talo de Couve (FTC) e Farinha de Talo de Espinafre (FTE) ricas em fibra alimentar. Cienc Tecnol Alim. 2010; 30(3):719-728.

26. Blaak EE. Carbohydrate quantity and quality and cardio-metabolic risk. Curr Opin Clin Nutr Metab Care. 2016;19(4):289-93.

27. Santos AAO, Santos AJAO, Alves AR, Santana FC, Silva JV, Marcellini PS. Elaboração de biscoitos a partir da incorporação de produtos da mandioca e casca de maracujá (Passiflora edulis flavicarpa) na farinha de trigo. Sci Plena. 2011; 7(8):1-7.

28. Leoro MGV, Clerici MTPS, Chang YK, Steel CJ. Avaliação de índice glicêmico in vitro de um cereal matinal extrusado produzido com farinha de milho e farelo de maracujá orgânicos. Cienc Tecnol Alim. 2010; 30(4): 964-968.

29. Livesey G, Taylor R, Hulshoft T, Howlett J. Glycemic response and health: a systematic review and metaanalysis: relations between dietary glycemic properties and health outcomes. Am J Clin Nutr. 2008; 87(1):58-68.

30. Brasil. Agência Nacional de Vigilância Sanitária. Resolução RDC nํ216, de 15 de setembro de 2004. Regulamento Técnico de Boas Práticas para Serviços de Alimentação. Diário Oficial da União. 2004, 16 set; Seção 1.

31. Fontbonne A, Cesse EAP, Sousa IMC, Souza WVS, Chaves VLV, Bezerra AFB. Risk factor control in hypertensive and diabetic subjects attended by the Family Health Strategy in the State of Pernambuco, Brazil: the SERVIDIAH Study. Cad. Saúde Pub. 2013; 29(6):1195-1204.

32. Carvalho ALM, Leopoldino RWD, Silva JEG, Cunha CP. Adesão ao tratamento medicamentoso em usuários cadastrados no Programa Hiperdia no município de Teresina (PI). Rev C S Col. 2012; 17(7):1885-92.

33. Mildner-Szkudlarz S, Bajerska J, Zawirska-Wojtasiak R, Górecka D. White grape pomace as a source of dietary fibre and polyphenols and its effect on physical and nutraceutical characteristics of wheat biscuits. J Sci Food Agric. 2013; 93:389-395.

34. Bueno APMM, Klajn VM. Elaboração e aceitabilidade de biscoitos enriquecidos com aveia e farinha de bagaço de uva. Braz. J. Food Technol. 2013; 16(1):68-72.

35. Soares Júnior MS, Caliari M, Torres MCL, Vera R, Teixeira JS, Alves LC. Qualidade de biscoitos formulados com diferentes teores de farinha de amêndoa de baru (Dipteryxalata Vog.). P Agrop Trop. 2007; 37(1):51-56.

36. Ajila CM, Leelavathi K, Prasada Rao, UJS. Improvement of dietary fiber content and antioxidant properties in soft dough cookies with the incorporation of mango peel powder. J Cereal Sci. 2008; 48: 319-326.

37. Aquino ACMS, Moés RS, Leão KMM, Figueiredo AVD, Castro AA. Avaliação físico-química e aceitação sensorial de biscoitos tipo cookies elaborados com farinha de resíduos de acerola. Rev Inst Adolfo Lutz. 2010; 69(3):379-86.

Recebido em maio de 2015.

Aprovado em agosto de 2016. 